\title{
AUTOMATIC FINITE ELEMENT MESHING OF PLANAR VORONOI TESSELLATIONS
}

\author{
Stefan Weyer \\ Universität Karlsruhe, Karlsruhe, Germany \\ Institut für Zuverlässigkeit und Schadenskunde im Maschinenbau \\ Andreas Fröhlich, Heinz Riesch-Oppermann ${ }^{\#}$ \\ Forschungszentrum Karlsruhe, Karlsruhe, Germany \\ Institut für Materialforschung II \\ Leon Cizelj, Marko Kovac \\ Institut “Jožef Stefan”, Ljubljana, Slovenia \\ Reactor Engineering Division
}

\begin{abstract}
The concept of Voronoi tessellation has recently been extensively used in materials science, especially to model the geometrical features of random microstructures like aggregates of grains in polycrystals, patterns of intergranular cracks and composites. Solution of the underlying field equations usually requires use of numerical methods such as finite elements.

The framework for automatic generation of quadrilateral finite element meshing of planar Voronoi tessellation is proposed in the paper, resulting in a powerful set of tools to be used in the rather wide field of micromechanics. As far as feasible, the implementation of features built in commercially available mesh generators was pursued. Additionally, the minimum geometric requirements for a "meshable" tessellation are outlined.
\end{abstract}

Special attention is given to the meshes, which enable explicit modelling of grain boundary processes, such as for example contact (closure of cracks) or friction between grains. This is inline with numerical examples, which are oriented towards the fracture mechanics, in particular to the development of intergranular microcracks and/or their impact on the effective behaviour of the polycrystal.

The examples were evaluated using the commercially available general-purpose finite element code ABAQUS. The usual continuum mechanics based numerical methods and boundary conditions were safely applied to aggregates of randomly oriented polycrystals with anisotropic elastic material behavior as computational domains.

Keywords: Voronoi tessellation, polycrystal, representative volume element, effective material properties, finite element method, stress corrosion cracking

\footnotetext{
\# Corresponding author. Tel. +49/7247/82 4155 Fax +49/7247/822347

E-mail: riesch-oppermann@imf.fzk.de (H. Riesch-Oppermann)
} 


\section{INTRODUCTION}

A Voronoi tessellation represents a cell structure constructed from a Poisson point process by introducing planar cell walls perpendicular to lines connecting neighbouring points. This results in a set of convex polygons/polyhedra embedding the points and their domains of attraction, which completely fill up the underlying space (an example is given in Fig. 2a). A survey about mathematical foundations and a variety of applications in different fields of science can be found for example in [1] or [2].

The concept of Voronoi tessellation has recently been extensively used in materials science, especially to model random microstructures like aggregates of grains in polycrystals, patterns of intergranular cracks and composites. Some examples of applications are calculation of properties of damaged [3] and non-damaged polycrystals [4], characterisation of fibrereinforced composites [4] simulation of microcrack nucleation and propagation in creep [6], [7], thermal fatigue [8] and intergranular stress-corrosion [9].

The applications of Voronoi tessellation are however not restricted to mechanical problems. Among others, simulation of magnetisation process in microstructures was performed [10]. Another possible application is to compute domain switching in ferroelectrica, as has been done with three-dimensional finite element simulation, but only with one cuboid element for each grain in [11].

In the above examples, the Voronoi tessellation was applied to model the geometrical features of the problems. In the examples it is necessary to solve the underlying field equations. In some special cases, satisfactory use of analytical [12] and empirical [8] solutions was feasible. Nevertheless, use of analytical or empirical approaches could severely limit the complexity of the analysis.

Therefore, use of general numerical methods such as for example finite or boundary elements may be necessary to obtain more accurate results. Examples of such applications are available:

- Simplified models, such as for example [13]: rigid grains, represented by Voronoi tessellation are connected to their neighbours with springs representing grain boundary conditions;

- 2-D finite element analysis, such as for example meshing of hexagonal grains with randomly moved vertices in [14];

- 3-D finite element analyses. Typically, the published analyses refrain from explicit modelling of grain boundaries. More common approach would be, for example, building a finite element mesh from cuboids and then assign the Gaussian point of elements to different grains, e.g., use different material properties [15], [16]. However, an example of using a 3-D Voronoi tessellation meshed by tetragonal elements is given in [10].

Application of finite element method requires discretization of the geometry into triangles, quadrilaterals or general convex polygons. The discretization with triangles is straightforward. This is because Voronoi tessellation is a dual of the Delaunay triangulation [2], which is a well-known partitioning method used in finite element mesh generation. On the other hand, the numerical quality of triangular finite elements is generally poor. Therefore, this topic will not be pursued further here. Another possibility is to use finite elements, which assume the 
shape of random Voronoi polygons (e.g., [17]). Obvious elegance of this approach unfortunately introduces some disadvantages, in particular, difficult if not impossible mesh refinements along grain boundaries. The quadrilateral elements were therefore chosen for further discussion.

In this paper, the framework for automatic generation of quadrilateral meshing of random grains is proposed, resulting in a powerful set of tools to be used in the rather wide field of micromechanics. The numerical examples are however oriented towards the fracture mechanics, in particular to the development of intergranular microcracks, their impact on the effective behaviour of the polycrystal and to the analysis of some stochastic properties of local stress fields in damaged and intact polycrystals.

Special attention is given to the of meshes, which enable modelling of grain boundary processes, such as for example contact (closure of cracks) or friction between grains. As far as feasible, the implementation of features built in commercially available mesh generators PATRAN [18] and CadFIX [19] was pursued. The current algorithms specialise in planar tessellation. This is because the computational efforts devoted to meshing and subsequent numerical solution of planar models easily outgrew the available resources. Meshing of about 1000 grains (e.g., about $0,1 \mathrm{~mm}^{2}$ of area assuming $30 \mu \mathrm{m}$ average grain size) requires some 35,000 to 40,000 finite elements. Generalisations to 3-D are nevertheless straightforward, pending further developments in computational hardware.

The performance of the proposed algorithms is demonstrated with two examples using the commercially available general-purpose finite element code ABAQUS [20]. All tessellations used in this paper were generated by the code VorTess [21]. 


\section{MESHING}

Interactive generation of a suitable finite element mesh of a large Voronoi tessellation (e.g., more than 100 grains-polygons) represents a rather challenging task. Moreover, a rather large number of consecutive analyses with different realisations of randomly shaped grain aggregates may be needed to obtain reliable estimates of probabilistic parameters. Therefore, an automatic meshing algorithm as described below is a necessity.

\section{1 "Meshable" Tessellation}

One of the basic requirements for reliable finite element analysis is suitable shape of the finite elements in the mesh. In the present meshing algorithms, we rely on planar quadrilateral elements. For them, the tolerable distortion from ideally square shape is limited [18]. The limits depend on the type of the analysis and the finite elements used.

Only a subset of all possible tessellations is meshable with quadrilateral elements. This subset of "meshable" tessellations has the following properties: (1) all angles within polygons exceed $30^{\circ}$ and (2) the maximum aspect ratio of the shortest and longest line within a single polygon is lower than a critical value. No strict limit has however been defined for the aspect ratio. We were able to mesh polygons with aspect ratio up to 1:500, provided that sufficiently small element sizes, and consequently very large number of elements (e.g., 20 or more per average polygon), were used.

The "meshable" tessellation, i.e. one with sufficiently small polygon aspect ratio and sufficiently large polygon angles, is simply obtained by the trial-and-error method. A series of realisations of tessellation is calculated and checked for the minimum requirements. The expected number of trials needed to obtain a suitable tessellation is plotted in Fig. 1 as a function of number of polygons and required aspect ratio. The expectations (lines) in Fig. 1 are obtained as fit to a finite set of successful trials, indicated by symbols. Fig. 1 suggests that about $10^{6}$ trials will be needed to obtain a tessellation with 1000 points with maximum aspect ratio less than 1:100. The selection of "meshable" tessellations does not affect typical statistical tessellation properties and serves only to facilitate the meshing procedure.

The bias introduced in the analysis by selecting only "meshable" tessellations is judged to be small compared to the error caused by the 2-D approximation of grain structure. This was further supported by numerical analysis of variances of the cell areas of the complete set of all and the reduced set of "meshable" Voronoi tessellations, which turned out to be approximately equal [4]. 


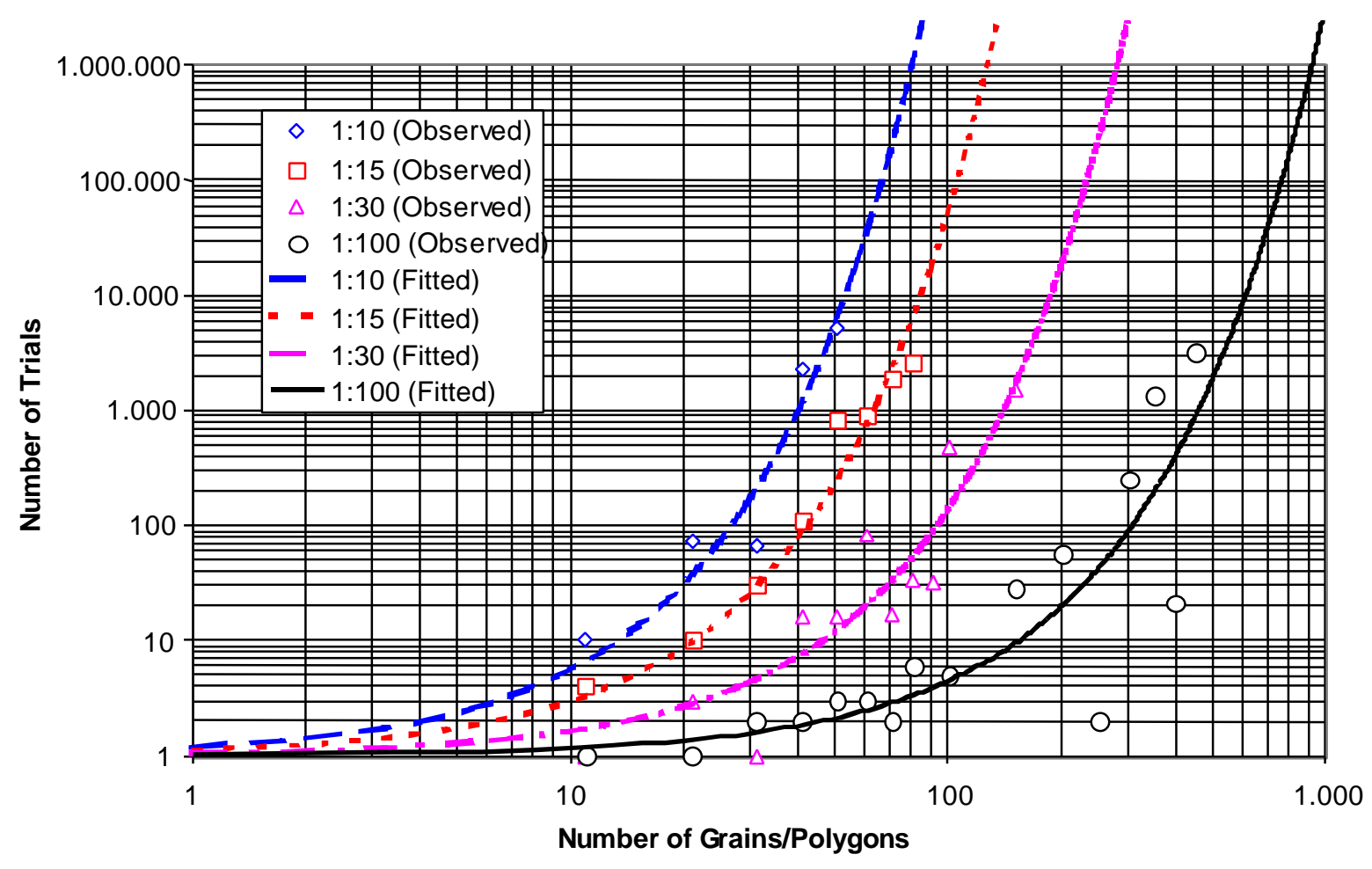

Fig. 1 Expected number of trials required to get a "meshable" tessellation.

\subsection{Quadrilateral meshing of Voronoi polygons}

The meshing strategy adopted in this paper is to mesh each grain as a separate body. This creates very useful framework for keeping track of the individual grains (to assign for example different random orientation of the crystallographic axes to each grain) and individual grain boundaries (e.g., to define possible and trace actual paths of intergranular cracks).

The geometry of each Voronoi polygon is described in terms of its vertices. These geometrical data are transferred via an interfacing computer code to the selected mesh generator (PATRAN, CadFIX) to form points (vertices), lines (polygon boundaries, grain boundaries) and surfaces (polygons, grains).

The mesh seed at a line (polygon boundary) of length $l$ is calculated as $l / e_{\min }$, where $e_{\min }$ represents the desired minimal length of the element edge. The shortest line in the tessellation gives an upper bound for $e_{\text {min }}$. The selection of $e_{\min }$ may also depend on the desired numerical accuracy of the analysis and the size of the problem. This proceeding assures that meshes fit together at the borders of neighbouring polygons. To get a finer mesh at the vertices, a variable mesh seed can be introduced which increases towards the middle part of the grain.

Meshing of the tessellation is at this stage performed automatically by the selected mesh generator (see Fig. 2). 

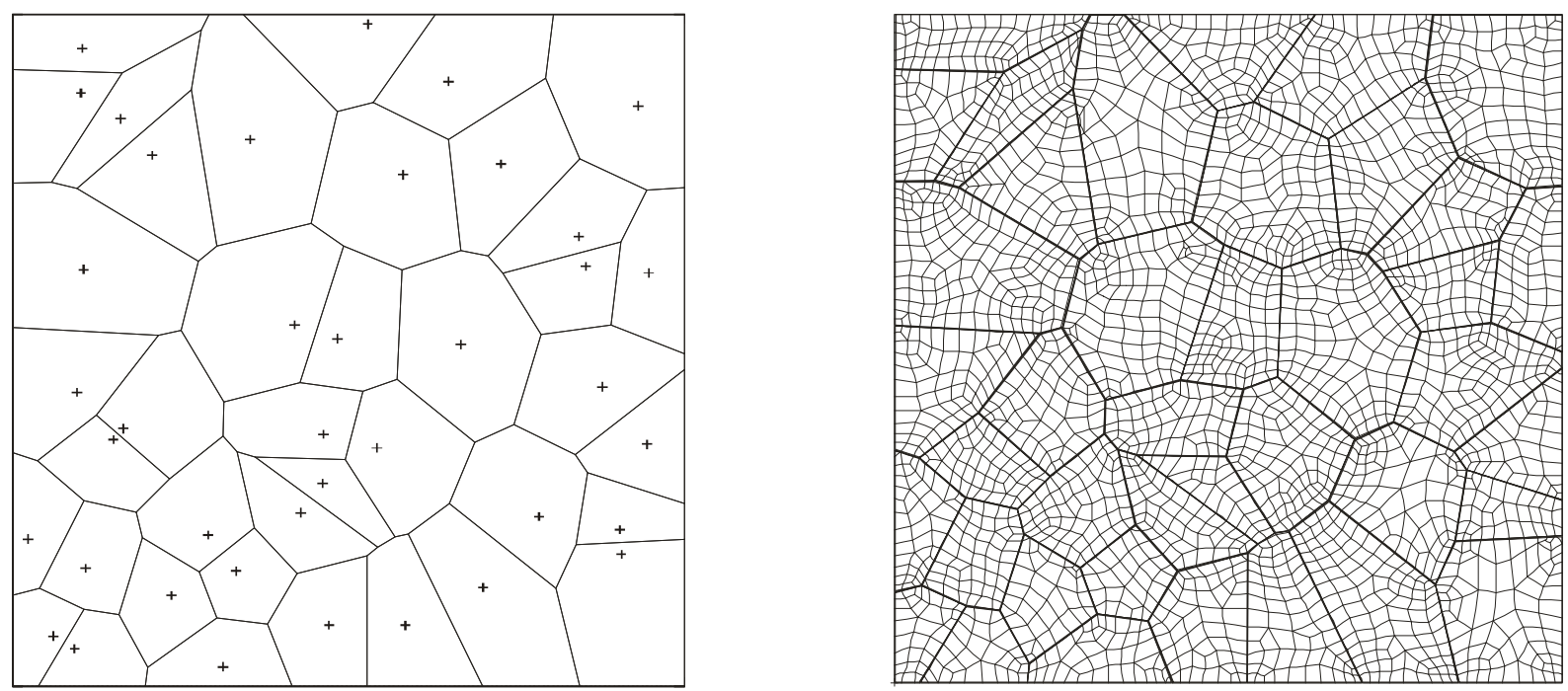

Fig. 2 a) Voronoi tessellation with 40 cells and the respective Poisson points. b) Finite element mesh for this tessellation.

\subsection{Mesh refinements at grain boundaries}

Grain boundaries may be a source of discontinuities originating from processes within the grains and/or at the grain boundary level due to the anisotropy of the grains and the random orientation of the crystallographic axes. Mesh refinement in grain boundary regions contributes significantly to the convergence and/or reliability of finite element solution. This section describes an advanced algorithm, developed to generate a refined mesh along all grain boundaries in the tessellation (Fig. 3)[32].

1. The number of requiered elements $A$ along each grain boundary surface is given according to

$$
A=3 \min _{n \in N}\left|\frac{l}{3 n}-e_{\min }\right| .
$$

As $A$ depends only on the length $l$ of the grain boundary, the compatibility of meshes of neighbouring grains is ensured. The value of $n$ has to be chosen in a way that $A$ becomes minimal.

2. The vertices of each polygon are projected by a distance of $2 e_{\min }$ along the centreline of the angle. An inner polygon is created with these projected vertices. This imposes an upper bound of $e_{\min }$ due to the requirement, that both inner and outer polygon are convex with the same number of vertices. The mesh with the lowest number of elements is obtained by selecting $e_{\min }$ at about $95 \%$ of this upper bound. Increasing the required minimal length for "meshable" tessellations may further reduce the number of elements.

3. All inner polygons are meshed as described in Section 2.2 .

4. The area between the outer and inner polygon is divided into a series of quadrilateral surfaces. Those surfaces are meshed with four elements each, so that the element length at the boundary of the inner polygon is three times higher than at the boundary of the outer polygon (see shaded area in Fig. 3a). This is possible, as $A$ is divisible by three. 
5. Sharp angles between the vertices of the Voronoi polygons may cause severe distortion of elements in the vicinity of vertices. To avoid such problems, a special strategy for meshing such corners was developed:

- We calculate the distances of the projected inner vertex to the original vertex and to every third of the neighbouring nodes on the adjoining grain boundaries (full circles in Fig. 3).

- If the original vertex leads to the smallest distance the angle is big enough and no special treatment is needed. Otherwise an additional surface is created between the original vertex, the inner vertex and the nodes located at the two adjoining outer boundaries with minimal distance to the original vertex.

- The additional surface is meshed by the selected mesh generator and results in quite regularly shaped elements in this critical region.

The modular structure of the above algorithm is also very easily applied in other cases. For example, some problems may require mesh refinements only at vertices. In such cases it is possible to refine mesh only in the vicinity of the vertices and mesh the surfaces along the borders with one element instead of four. This may be reasonable while modelling for example contact with friction, since the numerical effort increases drastically for each additional contact element at grain boundaries. With slight modifications, the above algorithm may be also used to mesh collapsed finite elements (crack tip singularities).

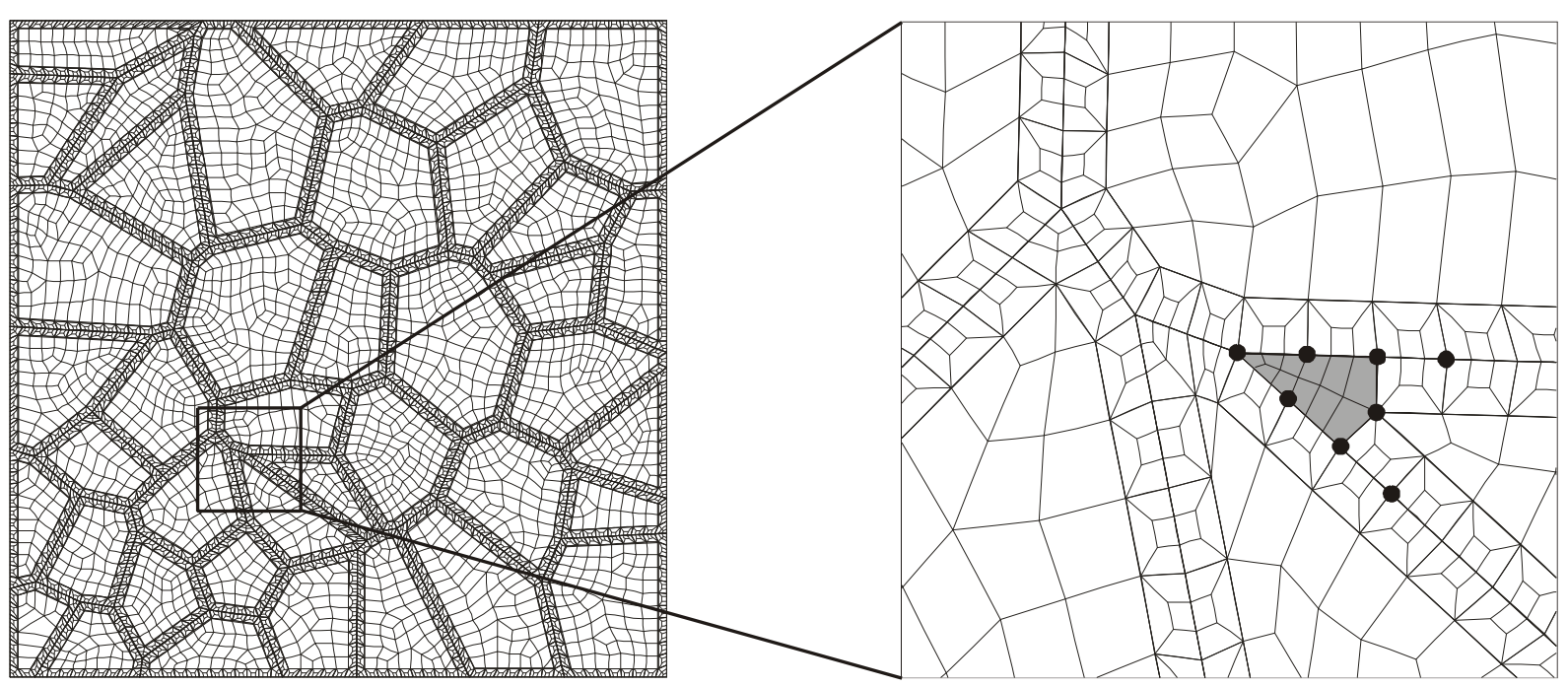

Fig. 3 Finite element mesh with mesh refinement at grain boundaries.

\section{$2.4 \quad$ Mesh connectivity at grain boundaries}

Grains, meshed as independent bodies, can be connected together in a variety of ways. The simplest procedure collapses the coincident nodes on grain boundaries. In some applications (e.g., crack closure with or without friction and sliding of grain boundaries in creep damage) it is useful to maintain the independence of the grain boundary nodes and connect them with some grain boundary specific models. Some possibilities would be:

- Gluing the adjoined grains by a series of constraints. This may for example enable the finite element solver to distinguish between failed and intact grain boundaries;

- Introducing the contact or interface elements between adjoined different grains. This may enable simulation of complex material behaviour at the grain boundaries [4]. 
The above approaches are applicable to sections 2.2 and 2.3 but lead to substantial increase in the computational effort required for the finite element solution.

\section{$2.5 \quad$ LOADING AND BOUNDARY CONDITIONS}

In a real polycrystal the stress and strain fields are affected by the random grain structure. If a certain grain aggregate within the material is selected, the boundary conditions of that aggregate are unknown. In micromechanics, boundary conditions are selected in a way that certain energy requirements are fulfilled. This leads to three classes of boundary condition which are characterized by either imposed homogeneous stresses or strains or by selection of periodic arrangements.

According to Hill [22] the homogeneous strain and stress boundary conditions lead to upper and lower bound limits for effective material properties.

An example of potential variability in results obtained by the use of different boundary conditions is shown in Fig. 7b. It is however to be noted that the difference is levelling out with increased number of analysed grains. Therefore, the usual stress or displacement controlled boundary conditions can be safely applied to a computational domain, which is large enough to be isotropic in a macroscopic sense. 


\section{NUMERICAL EXAMPLES}

This section describes the basic ideas and results of two applications of Voronoi tessellation, which relied on finite element meshing as described above.

\subsection{Local Fields and Effective Properties of Polycrystals}

The effective material properties (e.g. Young's modulus, shear modulus) are calculated from the parameters of a single crystal. Only a brief description of the method is given hereafter for completeness. Reader is referred to [23] for additional details.

An important application of the method proposed in [23] is estimation of the scatter of the stress- and strain fields at the micro scale. This is of special importance for example in the field of micro systems technology, where the length of components is in the order of a few grains. Of course, the anisotropy and the scatter of the material parameters is of utmost importance there.

The investigations are based on the consideration of a representative volume element (RVE). The RVE represents a statistical representative microstructure of the material. The volume averaging of strain and stress field within RVE leads to effective or overall properties of the material at the macroscopic level.

The random grain structure of a polycrystal is modeled as discussed in Section 2 with the help of planar Voronoi tessellation. Each Voronoi polygon is considered as a grain with anisotropic elastic material behavior and a random orientation of the crystallographic axes. The orientation is specified with the well-known Euler angles $\psi, \theta$ and $\varphi$ which are assumed to be random variables. $\psi$ and $\varphi$ are uniformly distributed on $[0 ; 2 \pi[$ and $\theta$ has the probability density $\sin (\theta)$ on $[0 ; \pi[$. An investigation of necessary number of grains to obtain approximately isotropic material behavior was performed. Results can be found in [23]

Boundary conditions have to be imposed on the volume element to calculate the stress and strain fields. In micromechanical models homogeneous traction and displacement boundary conditions according to $\left.\sigma(\vec{x})\right|_{\partial V}=\sigma_{0} \cdot \vec{n}(\vec{x})$ and $\left.\vec{u}(\vec{x})\right|_{\partial V}=\varepsilon_{0} \cdot \vec{x}$ are frequently used.

\subsubsection{Local fields}

Fig. 4 shows the stress field $\sigma_{11}\left(x_{1}, x_{2}\right)$ at the micro scale for an alumina polycrystal. The elastic properties of the trigonal single crystal can be found in [24]. The random grain structure and the random orientation of the grains leads to a stochastic stress field with $\sigma_{11}^{\min }=256 \mathrm{MPa}$ and $\sigma_{11}^{\max }=406 \mathrm{MPa}$. The highest stress concentration and stress gradients are observed at the grain boundaries due to the discontinuous material transition. The scatter of the stress field is a measure for the localization at the microscale. For further modeling of local plastic deformation or damage initiation at the microscale this variation of the micro fields is crucial. 
Fig. 5 shows the probability density of the local stress component $\sigma_{11}$ for displacement and traction boundary conditions. Considerable scatter of the stress for the two selected boundary conditions can be observed.
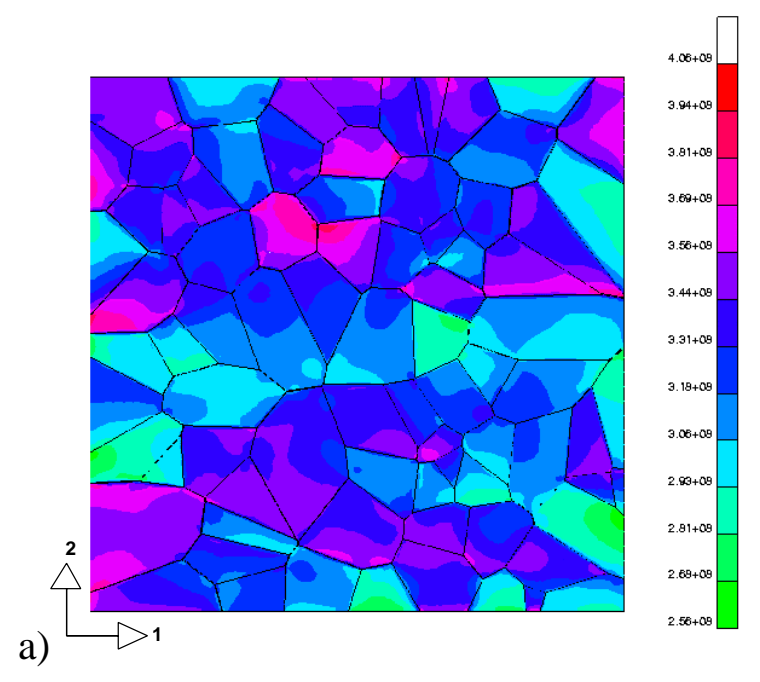

b)
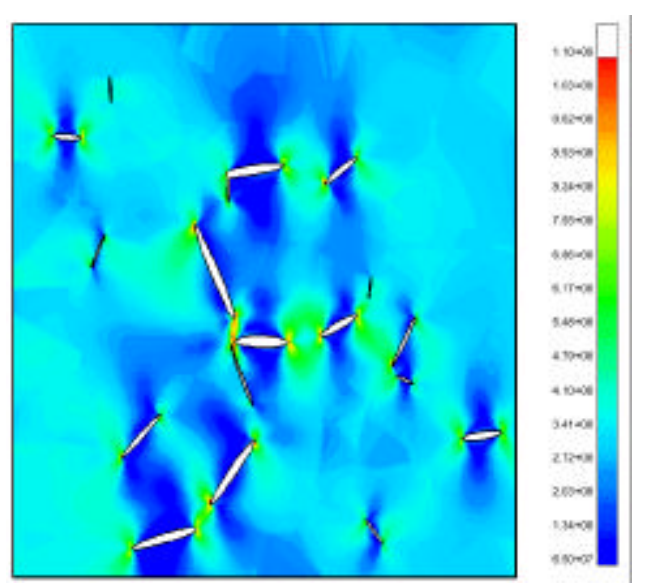

Fig. 4 Stochastic stress field $\sigma_{11}\left(x_{1}, x_{2}\right)$ a) $\mathbf{f = 0}$; displacement boundary conditions $\varepsilon_{11}^{0}=0.001$. b) $\mathbf{f}=\mathbf{0 . 0 5 3} ; \varepsilon_{22}^{0}=0.001$. The representative volume element consists of 80 grains. 

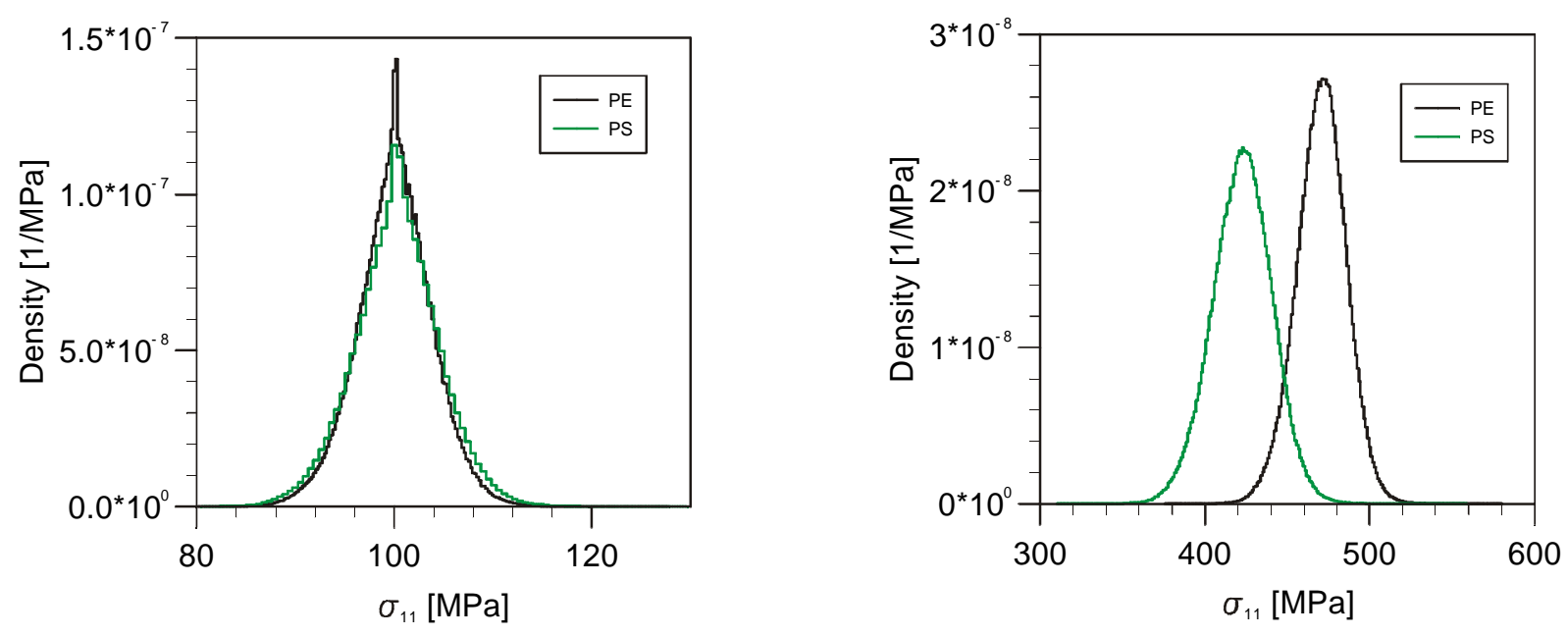

Fig. 5 Probability density of the stress $\sigma_{11}$ for plane stress (PS) and plane strain (PE). a) for traction and b) for displacement boundary conditions. The representative volume element consists of 500 grains and 50 realizations for the grain orientations are considered.

\begin{tabular}{|c|c|c|c|c|}
\hline & \multicolumn{2}{|l|}{ alumina } & \multicolumn{2}{|c|}{ barium titanate } \\
\hline model & plane strain & plane stress & plane strain & plane stress \\
\hline$\sigma_{11}[\mathrm{MPa}]$ & $100 \pm 2.9$ & $100 \pm 3.2$ & $100 \pm 10.4$ & $100 \pm 11.9$ \\
\hline$\sigma_{22}[\mathrm{MPa}]$ & $0 \pm 2.7$ & $0 \pm 2.8$ & $0 \pm 8.0$ & $0 \pm 8.1$ \\
\hline$\sigma_{12}[\mathrm{MPa}]$ & $0 \pm 1.5$ & $0 \pm 1.7$ & $0 \pm 4.8$ & $0 \pm 5.5$ \\
\hline
\end{tabular}

Table 1 Mean value and standard deviation of the local stress fields for traction boundary conditions $\sigma_{11}^{0}=100 \mathrm{MPa}$. The representative volume element consists of 500 grains and 50 realizations for the grain orientations are considered.

The mean values and the standard deviations of local stresses are shown in Table 1 for alumina and barium titanate. Plane strain and plane stress is considered. The single crystal values of barium titanate [24] show a larger deviation from the isotropic case than those of alumina. Therefore barium titanate shows higher fluctuations in the local stress fields. It can be seen that the standard deviation of the shear component is considerably smaller than the other stress components.

Fig. 6 a shows the probability density of the local stress $\sigma_{11}$ in a microcracked polycrystal as a function of microcrack density. The microcrack density $f$ is defined according to [25]. In the present application $f=0.1$ represents a rather severe density of microcracks (see also Fig. $4 \mathrm{~b}$, where $\mathrm{f}=0.053$ ). Due to the presence of microcracks one can observe a significant redistribution of stress. While the stress singularities at the crack tips clearly lead to higher maximum stresses, the unloading effect dominates the local field: the mean value of the stress decreases with increasing microcrack density as shown in Fig. 6 b. 

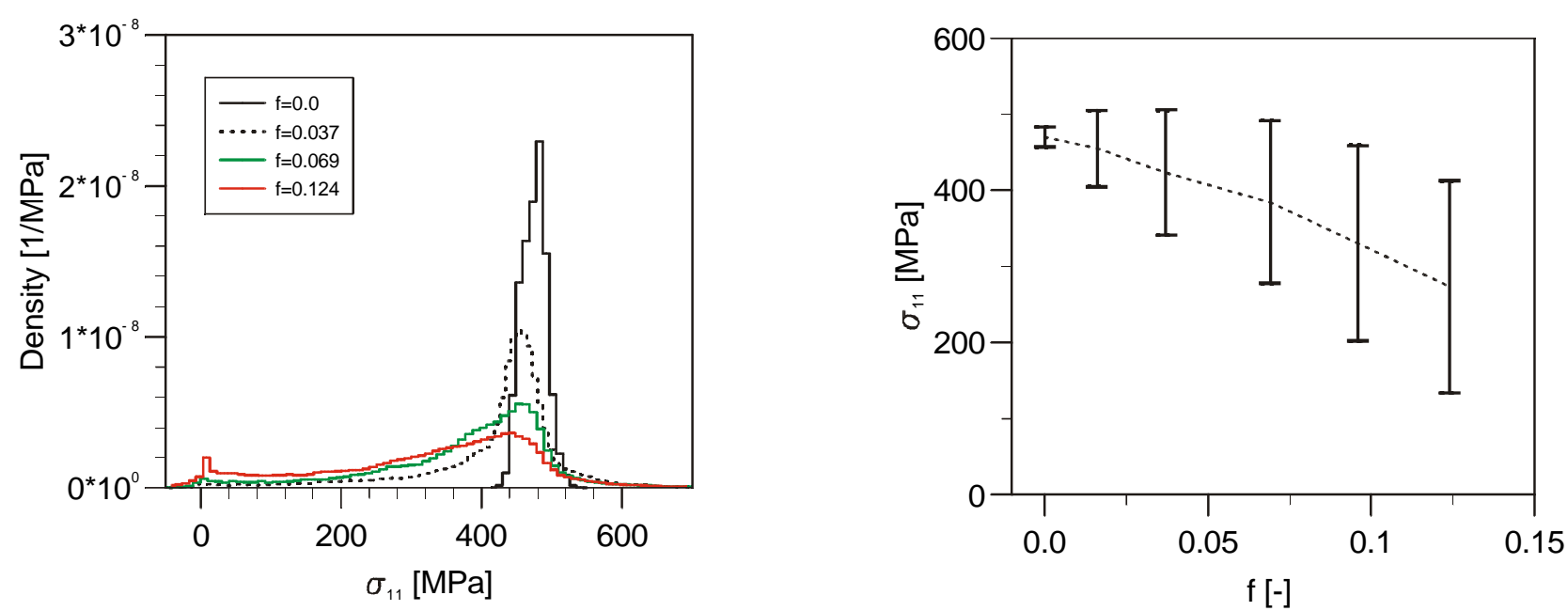

Fig. 6 a) Probability density and b) mean value and standard deviation of the local stress $\sigma_{11}$ in a microcracked polycrystal with 80 grains. Displacement boundary conditions are imposed.

These results are very important for the design of components in the micro system technology (E.g., micro planetary gear) with statistical methods [26].

\subsubsection{Effective Properties}

Several methods for determination of the effective properties of a heterogeneous microstructure from the single crystal values exist in literature. The well known Voigt [27] and Reuss [28] bounds on the effective properties are derived by averaging the stiffness and compliance tensors of the single crystals, respectively. The Hashin-Shtrikman variational principle [29] leads to narrower bounds for the effective properties, assuming statistical independence of grain orientations. The self consistent method [30] considers an ellipsoidal inclusion in an homogeneous, effective medium. It therefore takes into account a weak interaction between the micro constituents.

With our model we can consider a more realistic microstructure represented by a Voronoi tessellation, the local anisotropy of the grains and the interaction of the micro constituents.

The effective elasticity tensor $C^{*}$ is defined with volume averages of the local stress $\langle\sigma\rangle$ and strain field $\langle\varepsilon\rangle$ :

$$
\left\langle\sigma_{i j}\right\rangle=C_{i j k l}^{*}\left\langle\varepsilon_{k l}\right\rangle \text {. }
$$

Note that Eq. (2) is only an implicit definition of $C^{*}$. For the two-dimensional case we impose three linear independent boundary conditions to solve this equation in a unique way [23]. Due to the finite number of grains, a slight anisotropy remains present in $C^{*}$. This can either be neglected or eliminated by an averaging procedure over the full range of Eulerian angles. After calculating $C^{*}$ the effective Young's modulus, Poisson ratio and the shear modulus can be obtained in the ordinary way.

Using eq. (2), the effective material parameters for an $\mathrm{Al}_{2} \mathrm{O}_{3}$ polycrystal are calculated. The scatter of the Young's modulus is shown in Fig. 7a for Voronoi tessellations with 5 to 1000 
polygons (grains). For each number of grains, 50 different random orientations were simulated and displacement boundary conditions were imposed (plane strain).

The scatter of the Young's modulus decreases with increasing number of grains for the RVE. This behavior is typical, because of the decreasing influence of the orientation of a single grain for large RVE's.
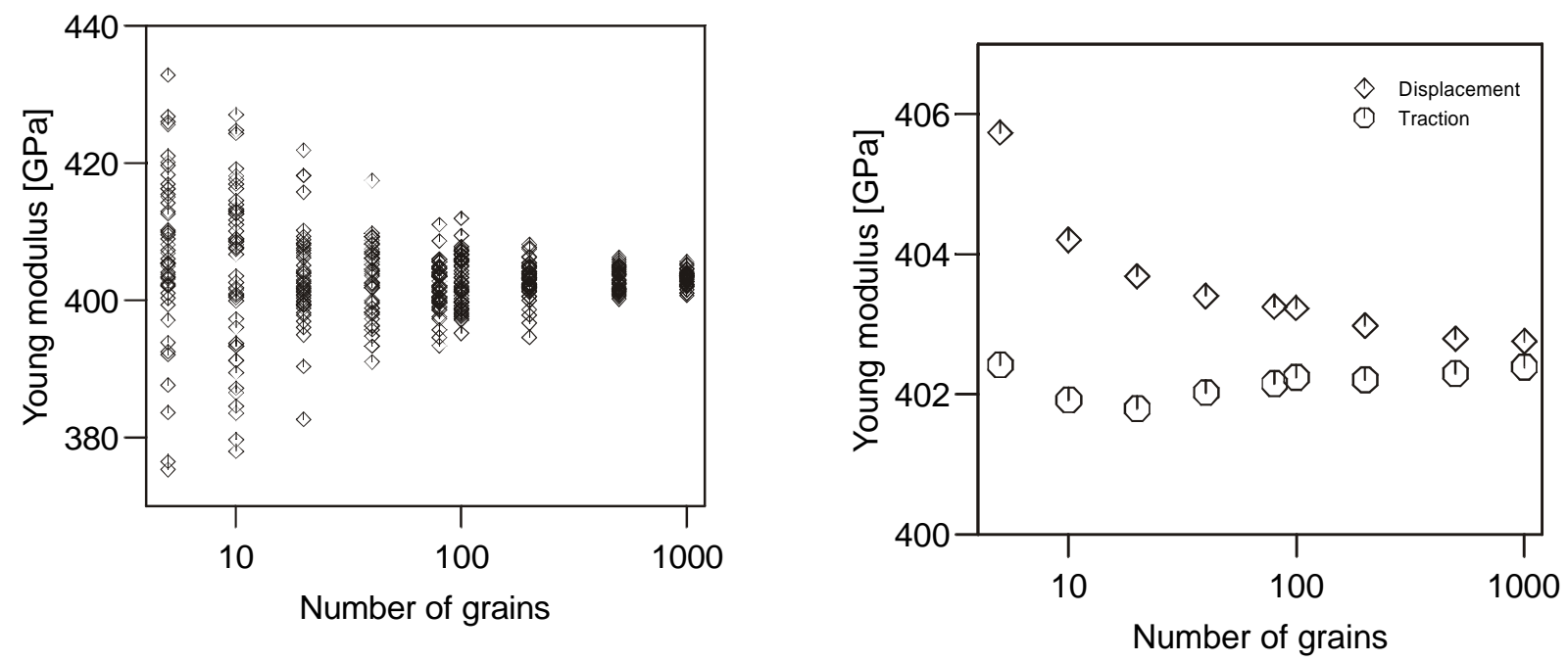

Fig. 7 a) Scatter of Young's modulus for 50 different random orientation simulations for each RVE from 10 to 1000 grains. b) Mean values of the Young's modulus for 50 calculations (plane strain). Higher and lower values are calculated with displacement and traction boundary conditions, respectively.

The mean values of the Young's modulus obtained with displacement boundary conditions are higher than those obtained by traction boundary conditions. This can be explained by artificially increased stiffness of the grains at the boundary. This increased stiffness is caused by the constraint effects from prescribed displacements at the boundary of the RVE. For an increasing number of grains those local effects vanish and the mean values of the Young's modulus converge to a limit value which may be interpreted as effective Young's modulus of the $\mathrm{Al}_{2} \mathrm{O}_{3}$ polycrystal.

The self-consistent method with a spherical grain shape predicts a Young's-modulus of 405.9 $\mathrm{GPa}$. The difference to the calculated values in Figure 5 is due to the fact that in the FEVoronoi model a more realistic microstructure, interaction between the grains and local anisotropy of the grains are fully taken into account.

\subsection{Development of Intergranular Stress Corrosion Cracks}

This section discusses the basic ideas and accuracy of method proposed to analyze the stress fields around tips of interacting cracks with complex random shapes. Crack initiation and propagation is caused by intergranular (stress) corrosion and/or creep crack growth. The fracture mechanics analysis is described in [31]. The shapes of the cracks and interaction effects are currently limited only with the underlying random grain structure (e.g., assuming development of intergranular crack growth). Some possible applications of this method may be found for example in [8], [9] and [14]. 


\subsubsection{Comments on meshing}

The Voronoi tessellation is used here to model the random grain structure. Further important assumptions are (1) the grain boundaries are only used as potential paths for the cracks and (2) the material in all grains is homogenous and isotropically elastic (for details see [8] and [9]). The failed grain boundaries are therefore modeled as cracks in an isotropic elastic continuum. This limitation is however not imposed by the method, but with our current understanding of micromechanical processes involved for example in stress corrosion cracking of Inconel 600 in hot water (see for example [9] and references therein). Such assumptions, combined with the use of domain integral methods, lead to reasonably accurate estimates of stress intensity factors with rather coarse isoparametric meshes (as shown in Fig. $2 b$, see [31] and references therein).

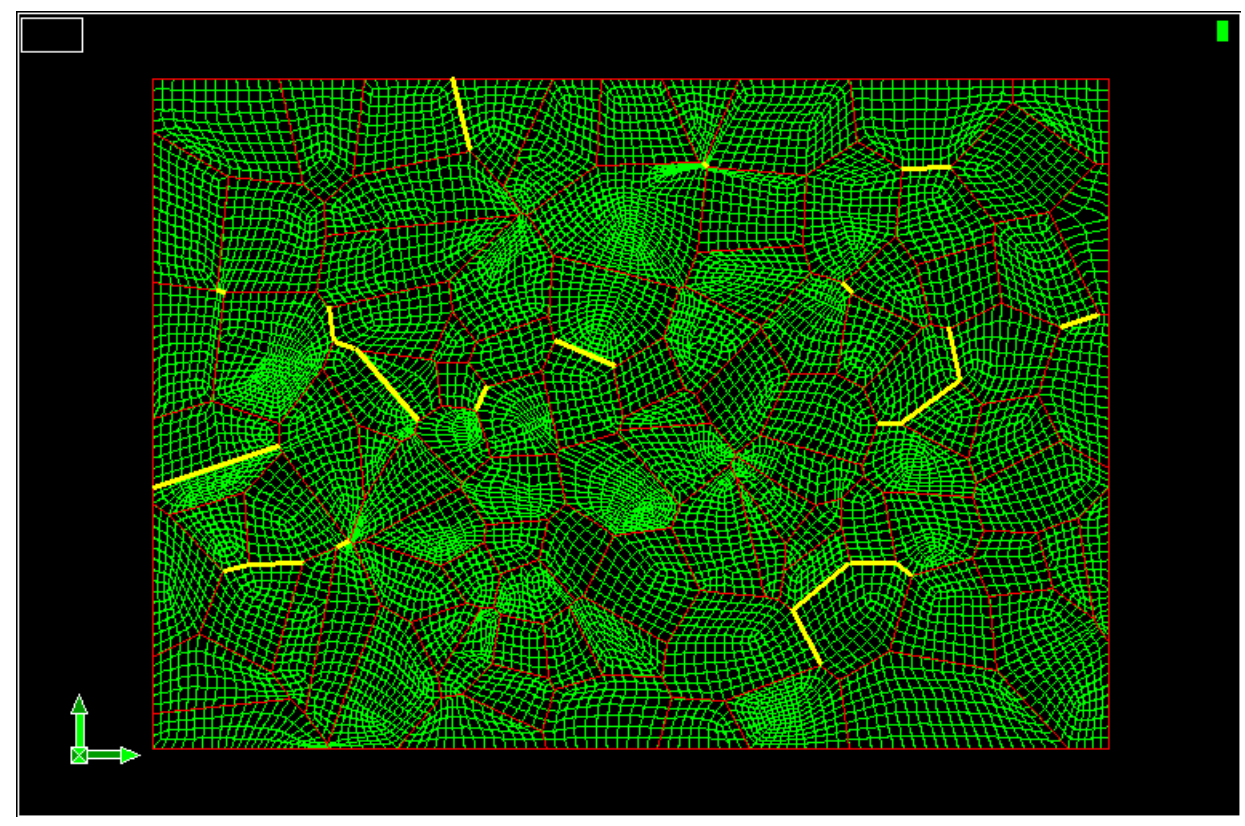

Fig. 8 Meshed grain structure with intergranular cracks (101 grain, 14 cracks).

Fig. 8 shows a typical Voronoi tessellation (101 grains, red lines) meshed by algorithm described in Section 2. Bold yellow lines denote the 14 cracks. The shapes of the 14 cracks were derived from the stochastic processes representing initiation and growth (for details see [9] and [31]) and should be seen as a shapshot from a simulated development of intergranular crack pattern [9]).

\subsubsection{Selected Results}

The model outlined in Fig. 8 was subjected to a remote bi-axial stress field with magnitudes 2 and 1 in directions of 1 and 2 (Fig. 9), respectively. The boundary conditions enforced the borders of the window to remain straight lines during the straining. The resulting stress field is depicted in (Fig. 9) in terms of von Mises equivalent stress. The areas with amplifications and reductions of the stresses due to the presence of cracks are clearly indicated. 
Fig. 10 gives a measure of accuracy of J-integral estimates at the tips of the straight cracks. The straight cracks are selected for comparison here since analytical solutions are available, at least without taking account of the interaction effects. The following conventions are used in Fig. 10 (see [31] for further details):

- J-integral error represents a relative error of the finite element estimate against appropriate analytical solution, which is assumed to have no error and does not account for any interaction effects;

- Relative size of typical element is simply the length of the crack divided by the length of finite element at the crack tip. Increasing the relative size of typical element would lead to increased numerical errors in J-integral, as shown by bold black lines in Fig. 10.

- STEP 0, 1 and 2 is used to distinguish between different complexities of the crack patterns. STEP 0 means that only simple straight cracks are present in the tessellation (e.g., crack initiation stage). STEP 1 and 2 include certain level of crack growth, which generates more complex patterns. Fig. 8 and Fig. 9 are snapshots from STEP 1.

The accuracy of J-integral estimates given in Fig. 10 and [31] is considered adequate. It should be noted here again that the zero error line assumes no interaction of cracks. However, it is clear from Fig. 9 that the straight cracks are small as compared to the other cracks in the pattern and that they are subjected to rather large interaction effects.

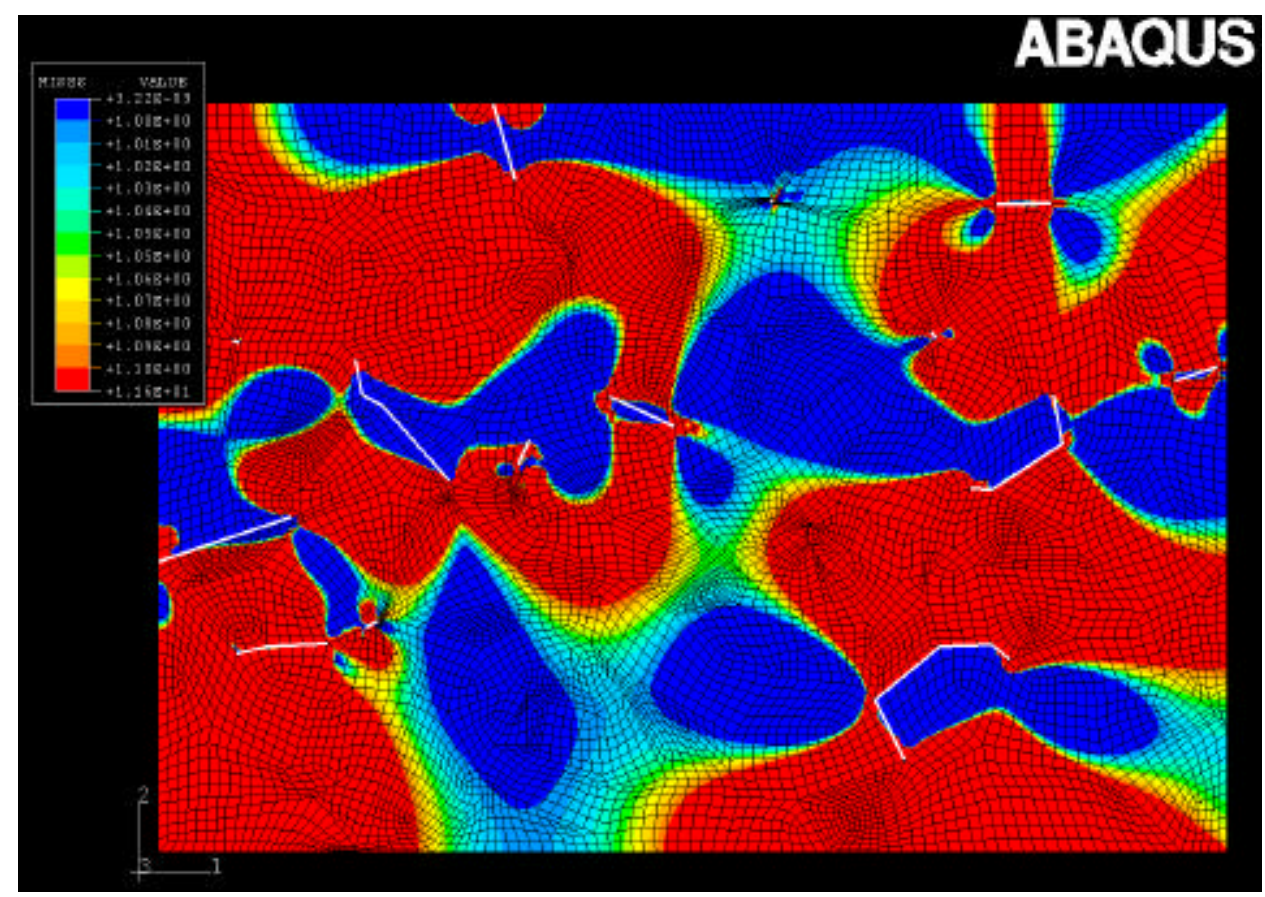

Fig. 9 Von Mises equivalent stress: areas with stress amplifications and reductions in red and blue, respectively. 


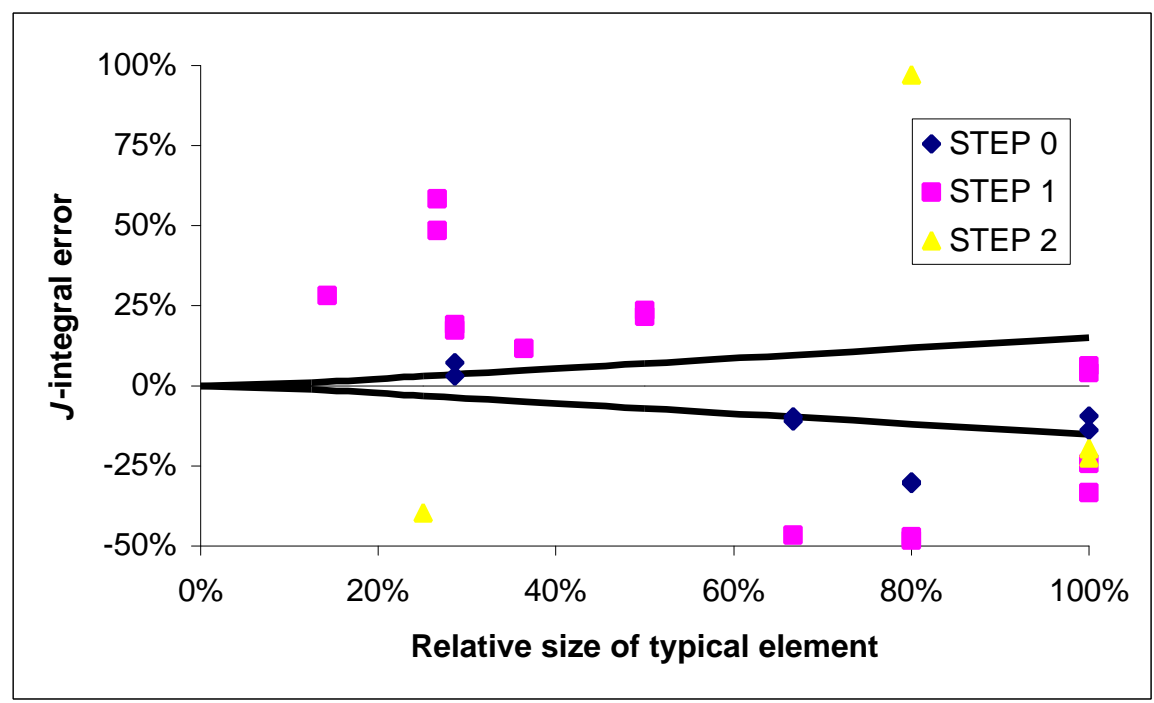

Fig. 10 Relative error of J-integral as compared to analytical results for straight cracks. 


\section{CONCLUSIONS}

The framework for automatic generation of quadrilateral meshing of Voronoi tessellation is proposed. Special attention is given to the meshes, which enable explicit modelling of physical processes on a grain boundary level, like stress- or corrosion-induced intercrystalline microcracking. Contact and friction effects between several grains can be modeled according to the capabilities of the available finite element code.

This is shown in the applications presented above, which are oriented towards fracture mechanics, in particular to the development of intergranular microcracks and/or their impact on the effective behaviour of the polycrystalline material.

The current algorithms specialise in planar tessellations. This is mainly because the computational efforts devoted to meshing and subsequent numerical solution of planar models easily outgrew the available resources. Additionally, the minimum geometric requirements for a "meshable" tessellation are outlined. As far as feasible, the implementation of features built in commercially available mesh generators was pursued.

Boundary condition based on homogeneously imposed stresses or displacements lead to upper and lower bounds in the elastic constants. With increasing number of grains, the bounds tend to coincide. Selection of a suitable RVE size can be based on admissible differences of upper and lower bounds.

The performance of the proposed framework is demonstrated with two examples using the commercially available general-purpose finite element code ABAQUS.

Future efforts will be focussed mainly in two directions. First, develop mesh refinements at the vertices of grains, aiming at reliable estimation of J-integrals at crack tip of essentially dissimilar materials (e.g., aggregate of randomly oriented anisotropic grains). An obvious long-term task is generalisation of the meshing algorithms to 3-D tessellations.

\section{ACKNOWLEDGEMENTS}

The authors are indebted to the Deutsche Forschungsgemeinschaft, Forschungszentrum Karlsruhe, Ministry of Science and Technology of Slovenia (Grant No. J2-7615) and International Office of the BMBF at Deutsches Zentrum für Luft- and Raumfahrt (Grant No. SLO-013/97). 


\section{REFERENCES}

[1] F. Aurenhammer, Voronoi diagrams - a survey of a fundamental geometric data structure, ACM Computing Surveys 23 (1991), 345-405.

[2] D. Stoyan, W.S. Kendall, J. Mecke, Stochastic geometry and its applications (2nd Edition), John Wiley \& Sons, Chichester, England (1995, Wiley Series in Probability and Statistics).

[3] S. Weyer, A. Brückner-Foit, A. Fröhlich, Overall Properties of Ceramics Subjected to Compressive Loading, International Conference on Engineering Ceramics and Structures, Ceramic Engineering and Science Proceedings 21 (2000), 101-107

[4] S. Weyer, Mikromechanisches Modell zur Berechnung effektiver Materialeigenschaften von geschädigten Polykristallen, Dissertation, Universität Karlsruhe, Germany (2001).

[5] T. Johansson, Analytische Beschreibung von Experimenten an faserverstärkten Keramiken zur Bestimmung von Grenzflächenparametern. VDI Reihe 18, No. 170, VDI-Verlag Düsseldorf, Germany (1995).

[6] E. van der Giessen, V. Tvergaard, Development of final creep failure in polycrystalline agregates, Acta Metall. Mater. 42 (1994), 959-973.

[7] P. Cannmo, An interface model based on damage coupled to slip and dilatation. Proceedings of the 12th Bienniel Conference on Fracture - ECF, Sep 14-18, 1998, Sheffield, U.K (1998), 957-962.

[8] E. Kullig, T. Johansson, A. Brückner-Foit, H. Riesch-Oppermann, D. Munz, T. Winkler, B. Michel, Ermittlung der Lebensdauerverteilung bei Thermoermüdung mit der Methoden der Stochastischen Geometrie, Report FZK-5692 (1996), Forschungszentrum Karlsruhe, Karlsruhe, Germany.

[9] L. Cizelj, H. Riesch-Oppermann, Modelling the Early Development of Secondary Side Stress Corrosion Cracks in Stream Generator Tube, Proceedings, International Symposium Contribution of Materials Investigation To the Resolution of Problems Encountered in Pressurized Water Reactors; Sep 14-18, 1998, Fontevraud, France, (1998), 583-594.

[10] H. Kronmüller, R. Fisher, M. Bachmann, T. Leineweber. Magnetization processes in small particles and nanocrystalline materials. Journal of Magnetism and Magnetic Materials 203(1999) 12-17.

[11] R. M. McMeeking, S.C. Hwang, A finite element model of ferroelastic polycrystals. Int. J. Solids and Structures. 36 (1999), 1541-1556.

[12] E. Kröner, Statistical Modelling. in J. Gittus and J. Zarka (eds): Modelling small deformations of Polycrystals. Elsevier Applied Science Publishers (1986), 229-291.

[13] Y. Toi, T. Kiyosure, Damage mechanics models for brittle microcracking solids based on threedimensional mesoscopic simulations, Engineering Fracture Mechanics, 50 (1995), 11-27.

[14] P. Onck, E. van der Giessen, Influence of microstructural variations on steady state creep and facet stresses in 2-D freely sliding polycrystals, International Journal of Solids and Structures, 34 (1997), 703726.

[15] F. Barbe, G. Cailletaud, S. Forest, F.E. Study of the Surface Effect in Polycrystalline Aggregates, Transactions of $15^{\text {th }}$ Int Conf on Structural Mechanics in Reactor Technology (SMiRT-15), Seoul, Korea, Aug. 15-20, 1999, XII-17-XII-28.

[16] N. Lippmann, Th. Steinkopff, S. Schmauder, P. Gumbsch, 3D-finite-element-modelling of multiphase elements, Computational Materials Science 9 (1997) 28-35.

[17] S. Ghosh, S. N. Mukhopadhyay, A material based finite element analysis of heterogeneous media 
involving Dirichlet tessellations, Computer Methods in Applied Mechanics and Engineering, 104 (1993), 211-247.

[18] MacNeal-Schwendler Corporation: MSC/PATRAN Users Guide Volume 2, Version 8.0.

[19] FEGS Ltd., CadFIX (FAM) Reference Manual (Version 2.1, release 7.0), Oakington, Cambridge, UK (1998).

[20] Hibbit, Karlsson, Sørensen. ABAQUS/Standard User's Manual, Version 5.8 (1998).

[21] H. Riesch-Oppermann, VorTESS, Generation of 2-D random Poisson-Voronoi Mosaics as Framework for the Micromechanical Modelling of Polycristalline Materials, Report FZKA 6325 (1999), Forschungszentrum Karlsruhe, Karlsruhe, Germany.

[22] R. Hill, The essential structure of constitutive laws for metal composites and polycrystals, J. Mech. Phys. Solids, 11 (1967) 79-95.

[23] S. Weyer, A. Fröhlich, A. Brückner-Foit, Micromechanical approach to calculate the overall properties of undamaged and damaged polycrystals, (submitted for publication).

[24] K.H. Hellwege, Numerical Data and Functional Relationships in Science and Technology, Group III: Crystal and Solid State Physics. Springer Verlag, Berlin (1979).

[25] B. Budiansky, R.J. O'Connell, Elastic moduli of a cracked solid, International Journal of Solids and Structures, 12 (1976) 81-97.

[26] A. Fröhlich, S. Weyer, D. Metz, O. Müller, A. Brückner-Foit, A. Albers, Investigations on the Reliability of FEA Calculations on the Microscale, $4^{\text {th }}$ International Conference on Modelling and Simulation of Microsystems, Hilton Head Island, SC, USA (March 19-21, 2001).

[27] W. Voigt, Über die Beziehung zwischen den beiden Elastizitätskonstanten isotroper Körper, Wiedemanns Annalen, 38 (1889) 573-587.

[28] A. Reuss, Berechnung der Fliessgrenze von Mischkristallen auf Grund der Plastizitätsbedingung für Einkristalle, Zeitung für angewandte Mathematik und Mechanik, 9 (1929) 49-58.

[29] Z. Hashin, S. Shtrikman, On some variational principles in anisotropic and nonhomogeneous elasticity, 10 (1962) 335-342.

[30] E. Kröner, Berechnung der elastischen Konstanten des Vielkristalls aus den Konstanten des Einkristalls 151 (1958) 504-518.

[31] M. Kovac, L. Cizelj, Numerical Analysis of Interacting Cracks in Biaxial Stress Field, Proc of Int Conf Nuclear Energy in Central Europe, Portorož, Slovenia, Sept. 1999, 259-266.

[32] A. Fröhlich, Mikromechanisches Modell zur Berechnung effektiver Materialeigenschaften von piezoelektrischen Polykristallen, Dissertation, Universität Karlsruhe, Germany (2001). 